\title{
Dirac Particle in External Non-Abelian Gauge Field
}

\author{
Bilel Hamil and Lyazid Chetouani \\ Département de Physique, Faculté des Sciences Exactes, Université Constantine 1, 25000 Constantine, Algeria \\ Correspondence should be addressed to Lyazid Chetouani; lyazidchetouani@gmail.com
}

Received 2 September 2013; Accepted 31 October 2013; Published 22 January 2014

Academic Editors: D. Chakrabarti, A. Koshelev, and B. Lucini

Copyright (C) 2014 B. Hamil and L. Chetouani. This is an open access article distributed under the Creative Commons Attribution License, which permits unrestricted use, distribution, and reproduction in any medium, provided the original work is properly cited.

\begin{abstract}
The Green function of a Dirac particle in interaction with a non-Abelian SU(N) gauge field exactly and analytically determined via the path integral formalism by using the approach so-called "global projection." The essential steps in the calculation are the choice of a convenient gauge (Lorentz gauge) and the introduction of two constraints, $\varphi=k x$ (related to space) and Grassmannian $\eta=k \psi$ (related to Dirac matrices). Furthermore, it is shown that certain selected equations obtained during the integrations can also be classically derived.
\end{abstract}

\section{Introduction}

At present the path integral has become indispensable, if we want to describe and to explain simply, some physical phenomena. The use of this tool is generalized to all domains of physics and this success is mainly due to the introduction of transformations which has allowed to solve the basic problems of the usual quantum mechanics, among others, the atom $\mathrm{H}$.

In nonrelativistic mechanics, we know that the form of the propagator is a sum over all possible paths, where at each path is assigned a weight which depends on the action of classical mechanics. With this standard form, the propagator has thus been determined for all soluble cases.

In the relativistic case, the insertion of spin (represented by matrices) and the limitation of the relativistic velocity are the difficulties encountered in the path integral formulation and a similar standard Feynman form is in principle, no longer valid.

However, from the viewpoint of calculation, the standard form seems more appropriate especially when it is question to determine the energies and wave functions for a system.

Thus, in the formulation path integral of Fradkin and Gitman [1] where the propagator has a similar expression to that standard of Feynman seems presently enough handy and easier to us when dealing to perform calculations. In this formulation, the motion is governed by a supersymmetric action and the parameterization used for the description two type of variables:

(i) bosonic (commuting) for the external movement;

(ii) fermionic (anticommuting or Grassmannian) for the internal movement.

This formalism has been tested on relativistic particles of spin $1 / 2$ and described by the Dirac equation moving under the action of fields having simple configurations such as the plane wave Volkov with or without the presence of an anomaly $[2,3]$, the wave which is nonclassical but quantified $[4,5]$, and other configurations [6]. Indirectly, the Dirac equation is solved via the determination of propagator by using the path integral approach in order to extract energies and wave functions of the Dirac particles.

Another configuration which has its importance in QCD and also in different domains of physics seemed useful to us in this paper to consider it in the path integral formalism. This configuration is that of a gauge field of type non-Abelian, which has not been considered anywhere in the literature, except the path integral formulation which can only be found in the book [7]. It is obvious that additional difficulties related to the non-Abelian group generators are introduced in the path integral formalism, in addition to that relating to spin of the particle. The control of this difficulty and their insertion in 
the formalism can be useful when the most complex problems in field theories are considered.

In this paper, we propose an alternative solution for a Dirac particle in which the gauge field is of type non-Abelian $\mathrm{SU}(\mathrm{N})$ and where the configuration is chosen the same as that used in [8], which allowed us to obtain analytically the solution of the Dirac equation. With this choice, it is shown that the propagator can also be analytically obtained by using simple changes.

The field in question has a particular configuration

(i) that is developable on the basis of generator $\left\{T^{a}\right\}_{a}$ of group $S U(N)$

$$
A_{\mu}=A_{\mu}^{a} T_{a}
$$

where $A_{\mu}^{a}$ are partial components and $T_{a}$, the generators of $S U(N)$ group which satisfy the commutative and anticommutative relations and normalization condition:

$$
\begin{gathered}
{\left[T_{a}, T_{b}\right]=T_{a} T_{b}-T_{b} T_{a}=i f_{a b}^{c} T_{c},} \\
{\left[T_{a}, T_{b}\right]_{+}=T_{a} T_{b}+T_{b} T_{a}=\frac{1}{N} \delta_{a b}+d_{a b}^{c} T_{c}} \\
\operatorname{tr}\left(T_{a} T_{b}\right)=\frac{1}{2} \delta_{a b},
\end{gathered}
$$

where $f_{a b}^{c}$ and $d_{a b}^{c}$ are the constants of the group $S U(N)$ (anti-symmetric and symmetrical, respectively, in all permutation of index).

In addition, the field is also chosen of type Volkov type; that is, it obeys the following properties:

(i) it is only a function product of $k x$, where $k$ is the 4vector wave such as

$$
k^{2}=k^{\mu} k_{\mu}=0,
$$

(ii) and it satisfies the condition of gauge of Lorentz

$$
\partial_{\mu} A^{\mu}=0, \quad k_{\mu} \dot{A}^{\mu}=0, \quad \text { or } \quad k A=0,
$$

where $\dot{A}=d A(k x) / d(k x)$ denotes the derivative with respect to $k x$.

In this paper, we use the so-called "global projection" of Alexandrou et al. [9], first by determining $G$ (where there are superfluous states) and then by projection, or thanks to the relationship between the two Green functions $G$ and $S$, we deduce $S$ (in order to obtain the physical states).

\section{Formulation of the Problem}

The Green function in question is solution of the following equation:

$$
\begin{array}{r}
\left(\gamma^{\mu}\left(i \partial_{\mu}-g A_{\mu}\right)-m\right) S\left(x, x^{\prime}\right)=\delta^{4}\left(x-x^{\prime}\right), \\
\mu=0,1,2,3,
\end{array}
$$

where $\gamma^{\mu}(\mu=0,1,2,3)$ are the usual matrices satisfying $\gamma^{\mu} \gamma^{\nu}+\gamma^{\nu} \gamma^{\mu}=2 g^{\mu \nu}$ and $g^{\mu \nu}=(+,-,-,-)$ is the metric tensor.

In the present paper, it is suggested to show, after simple changes, that the classical equations play an important role in the calculation of the propagator $S\left(x_{f}, x_{i}\right)$ for the particular form of field in question.

In configuration space, formally $S\left(x_{f}, x_{i}\right)$ is an element of matrix of the operator $S$, solution of

$$
\left(\gamma^{\mu} \mathscr{P}_{\mu}-m\right) S=I
$$

and the $S$ operator is equal to

$$
\begin{aligned}
S & =\frac{1}{O_{-}}=O_{+} \frac{1}{O_{-} O_{+}} \\
& =O_{+} G,
\end{aligned}
$$

where

$$
\begin{aligned}
& O_{ \pm}=\left(\gamma^{\mu} \mathscr{P}_{\mu} \pm m\right), \\
& \mathscr{P}_{\mu}=-p_{\mu}-g A_{\mu} .
\end{aligned}
$$

Knowing that $G$ and $S$ are connected by the following equation:

$$
S=\left(\gamma^{\mu} \mathscr{P}_{\mu}+m\right) G
$$

the Alexandrou et al. [9] approach is used to calculate $G$ (without the matrix $\gamma^{5}$ of the path integral formulation of Fradkin and Gitman).

It is easy to notice that

$$
\begin{gathered}
O_{-} O_{+}=\left\{p^{2}-m^{2}+g^{2}\left(\gamma^{\mu} A_{\mu}^{a} T_{a}\right)^{2}+2 g\left(p^{\mu} A_{\mu}^{a} T_{a}\right)\right. \\
\left.-i g\left(k_{\mu} \gamma^{\mu}\right)\left(\gamma^{\mu} \dot{A}_{\mu}^{a} T_{a}\right)\right\} ;
\end{gathered}
$$

the term $\left(\gamma^{\mu} A_{\mu}^{a} T_{a}\right)^{2}$ is equal to (see [8])

$$
\begin{aligned}
\left(\gamma^{\mu} A_{\mu}^{a} T_{a}\right)^{2}= & \left(\frac{\delta_{a b}}{N}+d_{a b}^{c} T_{c}+\frac{i}{2} f_{a b}^{c} T_{c}\right) \\
& \times\left(\eta_{\mu v}+\frac{1}{2}\left[\gamma^{\mu}, \gamma^{\nu}\right]\right) A_{\mu}^{a} A_{\nu}^{b} \\
= & \frac{A_{\mu}^{a} A_{a}^{\mu}}{2 N}
\end{aligned}
$$

after a calculation on the light cone.

Green's function $G$ to be determined is

$$
G\left(x_{f}, x_{i}\right)=-\frac{i}{2} \int_{0}^{\infty} d \lambda\left\langle x_{f}\left|e^{-(i \lambda / 2) \mathscr{H}}\right| x_{i}\right\rangle,
$$

where

$$
\begin{aligned}
\mathscr{H}(x, p)= & m^{2}-p^{2}-g^{2} \frac{A_{\mu}^{a} A_{a}^{\mu}}{2 N}-2 g\left(p^{\mu} A_{\mu}^{a} T_{a}\right) \\
& -i g\left(k_{\mu} \gamma^{\mu}\right)\left(\gamma^{\mu} \dot{A}_{\mu}^{a} T_{a}\right)
\end{aligned}
$$


is the Hamiltonian which governs the movement of the particle. To construct the path integral form of $G$, the usual procedure is used: the interval $\left[x_{i}, x_{f}\right]$ is subdivided into $N$ equal intervals of length $\Delta \tau=\lambda / N$, and then the closure relations are inserted

$$
\int|x\rangle\left\langle x\left|d^{4} x=1, \quad \int\right| p\right\rangle\langle p| d^{4} p=1
$$

with the scalar product for the change of base $|x\rangle \rightarrow|p\rangle$

$$
\langle x \mid p\rangle=\frac{1}{(2 \pi)^{2}} e^{i p x} .
$$

The continuous form of $G$ is

$$
\begin{aligned}
G\left(x_{f}, x_{i}\right)= & -\frac{i}{2} \mathscr{T} \int_{0}^{\infty} d \lambda \int D x \int D p \\
& \times \exp \left\{i \int_{0}^{\lambda}\left[p \dot{x}-\frac{1}{2} \mathscr{H}(x, p)\right] d \tau\right\},
\end{aligned}
$$

where $\mathscr{T}$-product is introduced due to the problem of order of the generators $T_{a}$ (operators) and $\gamma^{\mu}$ matrices do not commute. At this level, in order to eliminate the operators which are the generators $T_{a}$, the following procedure [7] is used:

(i) the generators are expressed by a bilinear relationship operators $\Gamma$

$$
T_{a}=\frac{i}{4} f_{a p}^{b} \Gamma_{b} \Gamma^{p}
$$

(ii) and the $\Gamma$ a relation of anticommutation is imposed

$$
\left[\Gamma_{b}, \Gamma_{p}\right]_{+}=\delta_{b p}
$$

So that $\Gamma$ becomes fermionic and $G$ is equal to

$$
\begin{aligned}
& G=-\frac{i}{2} \mathscr{T} \int_{0}^{\infty} d e \int D x \int D p \\
& \times \exp \left\{i \int_{0}^{\lambda}[p \dot{x}\right. \\
&+\frac{1}{2}\left(p^{2}-m^{2}+g^{2} \frac{A_{\mu}^{a} A_{a}^{\mu}}{2 N}\right. \\
&+\frac{i g}{2}\left(p^{\mu} A_{\mu}^{a} f_{a p}^{b} \Gamma_{b} \Gamma^{p}\right) \\
&\left.\left.\left.+\frac{g}{4}(k \gamma)\left(\gamma^{\mu} \dot{A}_{\mu}^{a} f_{a p}^{b} \Gamma_{b} \Gamma^{p}\right)\right)\right] d \tau\right\} .
\end{aligned}
$$

The elimination of operators is as follows: first anticommuting (Grassmann) variables are introduced using the following identity [1]:

$$
\begin{aligned}
& \mathscr{T} \exp \left\{\int_{0}^{\lambda} d \tau F(\Gamma(\tau))\right\} \\
& =\exp \left\{F\left(\frac{\delta}{\delta \varkappa_{a}}\right)\right\} \mathscr{T} \exp \left\{\int_{0}^{\lambda} \varkappa_{a} \Gamma^{a} d \tau\right\}_{x=0} ;
\end{aligned}
$$

(i) then the $\mathscr{T}$-product is removed by means of the following formula:

$$
\begin{aligned}
\mathscr{T} \exp \left\{\int_{0}^{\lambda} \varkappa_{a} \Gamma^{a} d \tau\right\}= & \exp \left(\Gamma^{a} \frac{\partial}{\partial \vartheta^{a}}\right) \int_{\zeta(1)+\zeta(0)=0} \mathscr{D} \xi \\
& \times \exp \left\{\int_{0}^{\lambda}\left(-\xi^{a} \dot{\xi}^{a}+2 \chi^{a} \xi^{a}\right) d \tau\right\} .
\end{aligned}
$$

These two formulae are also applicable for the $\gamma^{\mu}$ : it suffices to make the following replacement $\left(\Gamma^{a}, \vartheta^{a}, \xi^{a}\right) \rightarrow$ $\left(\gamma^{\mu}, \theta^{\mu}, \Psi^{\mu}\right)$.

Thus, Green's function takes the following form:

$$
\begin{aligned}
G= & -\frac{i}{2} \exp \left(\gamma^{\mu} \frac{\partial}{\partial \theta^{\mu}}+\Gamma^{a} \frac{\partial}{\partial \vartheta^{a}}\right) \int_{0}^{\infty} d \lambda \int D x \int D p \\
& \times \int \mathscr{D} \Psi \mathscr{D} \xi \\
& \times \exp \left\{i \int_{0}^{\lambda}[p \dot{x}+i \Psi \dot{\Psi}+i \xi \dot{\xi}\right. \\
& \left.\left.\quad-\frac{1}{2} \mathscr{H}(x, p, 2 \xi+\vartheta, 2 \Psi+\theta)\right]\right\}_{\theta=9=0}
\end{aligned}
$$

Let us make the change $(\xi, \Psi) \rightarrow(\zeta, \psi)$ defined by

$$
\begin{aligned}
& 2 \zeta(\tau)=2 \xi(\tau)+\vartheta \\
& 2 \psi(\tau)=2 \Psi(\tau)+\theta ;
\end{aligned}
$$

Green's function, to calculate in the global case, takes the following form:

$$
\begin{aligned}
G= & -\frac{i}{2} \exp \left(\gamma^{\mu} \frac{\partial}{\partial \theta^{\mu}}+\Gamma^{a} \frac{\partial}{\partial \vartheta^{a}}\right) \int_{0}^{+\infty} d \lambda \\
& \times \int D x D p \int_{A} \mathscr{D} \psi \int_{B} \mathscr{D} \zeta \\
& \times \exp \left\{i \int_{0}^{\lambda}[p \dot{x}+i \zeta \dot{\zeta}+i \psi \dot{\psi}\right. \\
& +\frac{1}{2}\left(p^{2}-m^{2}+g^{2} \frac{A_{\mu}^{a} A_{a}^{\mu}}{2 N}\right. \\
& +2 i g\left(p^{\mu} A_{\mu}^{a} f_{a p}^{b} \zeta_{b} \zeta^{p}\right) \\
& \left.\left.+4 g(k \psi)\left(\psi^{\mu} \dot{A}_{\mu}^{a} f_{a p}^{b} \zeta_{b} \zeta^{p}\right)\right)\right] d \tau \\
& +(0) \psi(\lambda)+\zeta(0) \zeta(\lambda)\}_{\theta=9=0}
\end{aligned}
$$


where the new domain of integration is

$$
\begin{aligned}
& A=\{\psi(\tau): \psi(\lambda)+\psi(0)=\theta\}, \\
& B=\{\zeta(\tau): \zeta(\lambda)+\zeta(0)=\vartheta\} .
\end{aligned}
$$

Let us proceed to practical calculation of $G$.

\section{Determination of Green Function}

As the four-potential $A_{\mu}^{a}$ depends only on $k x$, we put $k x=$ $\varphi$ and then the variable $\varphi$ is rendered independent of $x$ by introducing the following identities:

$$
\begin{aligned}
& \int d \varphi_{f} \int d \varphi_{i} \delta\left(\varphi_{i}-k x_{i}\right) \int D \varphi D p_{\varphi} \exp \left\{i \int_{0}^{\lambda} p_{\varphi}(\dot{\varphi}-k \dot{x}) d \tau\right\} \\
& =1 .
\end{aligned}
$$

Then, by making the shift $p_{\mu} \rightarrow p_{\mu}+k_{\mu} p_{\varphi}$, it becomes possible to integrate over $x$ and $p$, the integrations over $x$ can be easily performed and give $\delta(\dot{p})$, and the delta Dirac function expresses that the momentum is constant during the motion

$$
p_{1}=p_{2}=\cdots=p_{N+1}=p .
$$

Thus, $G$ is reduced to

$$
\begin{gathered}
G=-\frac{i}{2} \exp \left(\gamma^{\mu} \frac{\partial}{\partial \theta^{\mu}}+\Gamma^{a} \frac{\partial}{\partial \vartheta^{a}}\right) \int_{0}^{\infty} d \lambda \int \frac{d^{4} p}{(2 \pi)^{4}} \mathscr{M}(p) \\
\quad \times \int d \varphi_{f} d \varphi_{i} \delta\left(\varphi_{i}-k x_{i}\right) \int D \varphi \int D p_{\varphi} \int \mathscr{D} \psi \mathscr{D} \zeta \\
\quad \times \exp \left\{i \int_{0}^{\lambda}[i \psi \dot{\psi}+i \zeta \dot{\zeta}\right. \\
+\frac{1}{2}\left(4 g(k \psi)\left(\psi^{\mu} \dot{A}_{\mu}^{a} f_{a p}^{b} \zeta_{b} \zeta^{p}\right)\right. \\
\left.+g^{2} \frac{A_{\mu}^{a} A_{a}^{\mu}}{2 N}+2 i g\left(p^{\mu} A_{\mu}^{a} f_{a p}^{b} \zeta_{b} \zeta^{p}\right)\right) \\
\left.+p_{\varphi}(\dot{\varphi}+k p)\right] d \tau \\
+\psi(0) \psi(\lambda)+\zeta(0) \zeta(\lambda)\},
\end{gathered}
$$

where $\mathscr{M}(p)$ is given by

$$
\mathscr{M}(p)=\exp \left[i p\left(x_{f}-x_{i}\right)+\frac{i \lambda}{2}\left(p^{2}-m^{2}\right)\right] .
$$

The integrations on the $p_{\varphi}$ can be then performed

$$
\int D p_{\varphi} \exp \left[i \int_{0}^{\lambda} p_{\varphi}(\dot{\varphi}+k p) d \tau\right]=\delta(\dot{\varphi}+k p)
$$

and this show the solution path of

$$
\dot{\varphi}+k p=0,
$$

which is a line

$$
\varphi(\tau)=\varphi_{i}-k p \tau
$$

contributing mainly to the calculation of $G$.

It is useful to note that $A_{\mu}^{a}$ is now function of the variable $\tau$.

Similarly, by posing $\eta=k \psi$, the variables $\psi$ and $\eta$ become independent. By introducing the following identity:

$$
\begin{aligned}
& \int d \eta_{i} d \eta_{f} \delta\left(\eta_{i}-k \psi_{i}\right) \int D \eta \int D p_{\eta} \exp \left\{i \int_{0}^{\lambda} p_{\eta}(\dot{\eta}-k \dot{\psi}) d \tau\right\} \\
& =1
\end{aligned}
$$

where $\eta$ and $p_{\eta}$ are fermionic variables, expression of $G$ becomes

$$
\left.\begin{array}{rl}
G= & -\frac{i}{2} \exp \left(\gamma^{\mu} \frac{\partial}{\partial \theta^{\mu}}+\Gamma^{a} \frac{\partial}{\partial \vartheta^{a}}\right) \int_{0}^{\infty} d \lambda \int \frac{d^{4} p}{(2 \pi)^{4}} \mathscr{M}(p) \\
& \times \int d \varphi_{f} \delta\left(\varphi_{f}-\varphi_{i}+\lambda k p\right) \int d \varphi_{i} \delta\left(\varphi_{i}-k x_{i}\right) \\
& \times \int d \eta_{i} d \eta_{f} \delta\left(\eta_{i}-k \psi_{i}\right) \int D \eta \int D p_{\eta} \int \mathscr{D} \psi \mathscr{D} \zeta \\
& \times \exp \left\{i \int_{0}^{\lambda}[i \psi \dot{\psi}+i \dot{\zeta \zeta}\right. \\
& +\frac{1}{2}\left(g^{2} \frac{A_{\mu}^{a} A_{a}^{\mu}}{2 N}+2 i g\left(p^{\mu} A_{\mu}^{a} f_{a p}^{b} \zeta_{b} \zeta^{p}\right)\right. \\
+4 g \eta\left(\psi^{\mu} \dot{A}_{\mu}^{a} f_{a p}^{b} \zeta_{b} \zeta^{p}\right) & \left.+p_{\eta}(\dot{\eta}-k \dot{\psi})\right] d \tau \\
+\psi(0) \psi(\lambda)+\zeta(0) \zeta(\lambda)
\end{array}\right\}
$$

Now we replace the integration over $\psi$ by one over odd velocities $\omega$

$$
\psi^{\mu}(\tau)=\frac{1}{2} \int_{0}^{\lambda} \varepsilon\left(\tau-\tau^{\prime}\right) \omega^{\mu}\left(\tau^{\prime}\right) d \tau^{\prime}+\frac{\theta^{\mu}}{2},
$$

such that there are no restrictions on $\omega$ and the boundary conditions for $\psi$ are satisfied.

It appears thus to be a quadratic form of $\omega^{\mu}(\tau)$, and with the following change:

$$
\omega^{\mu}(\tau) \longrightarrow \omega^{\mu}(\tau)+i k^{\mu} \int_{0}^{\lambda} \varepsilon^{-1}\left(\tau-\tau^{\prime}\right) p_{\eta}\left(\tau^{\prime}\right) d \tau^{\prime},
$$


the Green function takes the following form:

$$
\begin{aligned}
& G=-\frac{i}{2} \exp \left(\gamma^{\mu} \frac{\partial}{\partial \theta^{\mu}}+\Gamma^{a} \frac{\partial}{\partial \vartheta^{a}}\right) \int_{0}^{\infty} d \lambda \int \frac{d^{4} p}{(2 \pi)^{4}} \mathscr{M}(p) \\
& \times \int d \varphi_{f} \delta\left(\varphi_{f}-\varphi_{i}+\lambda k p\right) \int d \varphi_{i} \delta\left(\varphi_{i}-k x_{i}\right) \\
& \times \int d \eta_{i} d \eta_{f} \int d p_{\eta_{i}} \int D \eta D p_{\eta} \int \mathscr{D} \omega \mathscr{D} \zeta \\
& \times \exp \{\zeta(0) \zeta(\lambda) \\
& +i \int_{0}^{\lambda}\left[\frac{-i}{2} \omega_{\mu} \varepsilon \omega^{\mu}+i \zeta \dot{\zeta}+p_{\eta} \dot{\eta}\right. \\
& +\frac{e}{2}\left(g^{2} \frac{A_{\mu}^{a} A_{a}^{\mu}}{2 N}+2 i g\left(p^{\mu} A_{\mu}^{a} f_{a p}^{b} \zeta_{b} \zeta^{p}\right)\right. \\
& +2 g \eta\left(\theta^{\mu} \dot{A}_{\mu}^{a} f_{a p}^{b} \zeta_{b} \zeta^{p}\right) \\
& \left.\times 2 g \eta\left(\dot{A}_{\mu}^{a} f_{a p}^{b} \zeta_{b} \zeta^{p}\right) \varepsilon \omega^{\mu}\right) \\
& \left.\left.+\frac{1}{\lambda} p_{\eta_{i}}\left(\eta_{i}+\frac{k(\omega-\theta)}{2}\right)\right] d \tau\right\}
\end{aligned}
$$

where the function $\delta$ is replaced by the exponential form

$$
\delta\left(\eta_{i}+\frac{k}{2}(\omega-\theta)\right)=\int d p_{\eta_{i}} \exp \left[i p_{\eta_{i}}\left(\eta_{i}+\frac{k}{2}(\omega-\theta)\right)\right] .
$$

Then, it is possible to perform the integration over the $p_{\eta}$. Again, it is appears that Dirac function

$$
\int D p_{\eta} \exp \left[i \int_{0}^{\lambda} p_{\eta} \dot{\eta} d \tau\right]=\delta(\dot{\eta})
$$

that is, the path of equation

$$
\dot{\eta}=0 \Longleftrightarrow \eta=\eta_{i}=\eta_{f}
$$

contributes mainly to the determination of $G$.

After rearrangement, the following standard form is obtained for the integral over velocities $\omega^{\mu}$

$$
\int \mathscr{D} \omega \exp \left\{\int_{0}^{\lambda}\left[\frac{1}{2} \omega_{\mu} \varepsilon \omega^{\mu}+\mathscr{J}_{\mu} \omega^{\mu}\right] d \tau\right\},
$$

where

$$
\mathscr{J}_{\mu}(\tau)=\frac{i}{2} k_{\mu} p_{\eta_{i}}+i g e \eta_{i} \int_{0}^{\lambda}\left(\dot{A}_{\mu}^{a} f_{a p}^{b} \zeta_{b} \zeta^{p}\right) \varepsilon\left(\tau^{\prime}-\tau\right) d \tau^{\prime} .
$$

As the result of the integration is

$$
\exp \left\{\frac{1}{2} \iint_{0}^{\lambda} \mathscr{J}_{\mu}(\tau) \varepsilon^{-1}\left(\tau-\tau^{\prime}\right) \mathscr{J}^{\mu}\left(\tau^{\prime}\right) d \tau d \tau^{\prime}\right\},
$$

and thanks to properties of the Volkov field, it is easy to show that

$$
\mathscr{J}_{\mu} \varepsilon^{-1} \mathscr{J}^{\mu}=0
$$

and that the integrations on $p_{\eta_{i}}$ is simply

$$
\int d p_{\eta_{i}} \exp \left[i p_{\eta_{i}}\left(\eta_{i}-\frac{k \theta}{2}\right)\right]=\delta\left(\eta_{i}-\frac{k \theta}{2}\right),
$$

that is, thus fixing $\eta_{i}=\eta_{f}=(k / 2) \theta$.

Now, Green's function has the following form:

$$
\begin{aligned}
G= & -\frac{i}{2} \exp \left(\gamma^{\mu} \frac{\partial}{\partial \theta^{\mu}}+\Gamma^{a} \frac{\partial}{\partial \vartheta^{a}}\right) \int_{0}^{\infty} d \lambda \frac{d^{4} p}{(2 \pi)^{4}} \mathscr{M}(p) \\
& \times \int d \varphi_{f} \delta\left(\varphi_{f}-\varphi_{i}+\lambda k p\right) \int d \varphi_{i} \delta\left(\varphi_{i}-k x_{i}\right) \int \mathscr{D} \zeta \\
& \times \exp \left\{i \int _ { 0 } ^ { \lambda } \left[i \zeta \dot{\zeta}+\frac{1}{2}\left(g(k \theta)\left(\theta^{\mu} \dot{A}_{\mu}^{a} f_{a p}^{b} \zeta_{b} \zeta^{p}\right)\right.\right.\right. \\
& +g^{2} \frac{A_{\mu}^{a} A_{a}^{\mu}}{2 N} \\
+\zeta(0) \zeta(\lambda)\}_{\theta=9=0} & \left.\left.+2 i g\left(p^{\mu} A_{\mu}^{a} f_{a p}^{b} \zeta_{b} \zeta^{p}\right)\right)\right] d \tau
\end{aligned}
$$

There are still integrations to be performed on variables related to generators of the group $S U(N)$.

Using the change $\zeta \rightarrow \xi$ defined by

$$
\zeta(\tau)=\xi(\tau)+\frac{1}{2} \vartheta
$$

then $G$ becomes

$$
\begin{aligned}
G= & -\frac{i}{2} \exp \left(\gamma^{\mu} \frac{\partial}{\partial \theta^{\mu}}+\Gamma^{a} \frac{\partial}{\partial \vartheta^{a}}\right) \int_{0}^{\infty} d \lambda \int \frac{d^{4} p}{(2 \pi)^{4}} \\
& \times \int d \varphi_{f} \delta\left(\varphi_{f}-\varphi_{i}+e k p\right) \int d \varphi_{i} \delta\left(\varphi_{i}-k x_{i}\right) \\
& \times \int \mathscr{D} \xi \exp \left\{i p\left(x_{f}-x_{i}\right)\right. \\
& \left.+\int_{0}^{\lambda}\left(i \xi \dot{\xi}-\frac{1}{2} \mathscr{H}(p, 2 \xi+\vartheta)\right) d \tau\right\}_{\theta=9=0},
\end{aligned}
$$

and let us return to two identities (21) and (20). 
After having eliminated the $\xi$ variables, the relationship between $T$ and $\Gamma$ can be reintroduced and Green's function takes the following form:

$$
\begin{aligned}
G= & -\frac{i}{2} \exp \left(\gamma^{\mu} \frac{\partial}{\partial \theta^{\mu}}\right) \int_{0}^{\infty} d \lambda \int \frac{d^{4} p}{(2 \pi)^{4}} \mathscr{M}(p) \\
& \times \int d \varphi_{f} \delta\left(\varphi_{f}-\varphi_{i}+\lambda k p\right) d \varphi_{i} \delta\left(\varphi_{i}-k x_{i}\right) \\
& \times \exp \left\{\frac { i } { 2 } \int _ { 0 } ^ { \lambda } \left(g^{2} \frac{A_{\mu}^{a} A_{a}^{\mu}}{2 N}-i g(k \theta)\left(\theta^{\mu} \dot{A}_{\mu}^{a} T_{a}\right)\right.\right. \\
& \left.\left.+2 g\left(p^{\mu} A_{\mu}^{a} T_{a}\right)\right) d \tau\right\}_{\theta=0},
\end{aligned}
$$

where the $\mathscr{T}$-product is omitted, since there is no order problem.

In order to reintroduce the $\gamma^{\mu}$ : the calculation of derivations is facilitated by using the following identity:

$$
\exp \left(\gamma^{\mu} \frac{\partial}{\partial \theta^{\mu}}\right) F(\theta)_{\theta=0}=F\left(\frac{\partial}{\partial \theta^{\mu}}\right) \exp \left(\theta_{\mu} \gamma^{\mu}\right)_{\theta=0}
$$

Then, after derivation, Green's function becomes

$$
\begin{aligned}
G= & \frac{-i}{2} \int_{0}^{\infty} d \lambda \int \frac{d^{4} p}{(2 \pi)^{4}} \mathscr{M}(p) \int d \varphi_{f} \\
& \times \int d \varphi_{i} \delta\left(\varphi_{i}-k x_{i}\right) \delta\left(\varphi_{f}-\varphi_{i}+e k p\right) \\
& \times \exp \left\{\frac{i g^{2}}{2 p k} \int_{\varphi_{i}}^{\varphi_{f}} \frac{A_{\mu}^{a} A_{a}^{\mu}}{2 N} d \varphi-\frac{i g}{p k}\right. \\
& \left.\times \int_{\varphi_{i}}^{\varphi_{f}}\left(\left(p^{\mu} A_{\mu}^{a}\right)-i\left(\frac{k \gamma}{2}\right)\left(\gamma^{\mu} \dot{A}_{\mu}^{a}\right)\right) T_{a} d \varphi\right\},
\end{aligned}
$$

where the two functions $\delta$ have been removed. Using the integral representation

$$
\delta\left(\varphi_{f}-\varphi_{i}+\lambda k p\right)=\int \frac{d p_{\varphi_{f}}}{2 \pi} \exp \left\{i p_{\varphi_{f}}\left(\varphi_{f}-\varphi_{i}+\lambda k p\right)\right\}
$$

and by changing we obtain

G

$$
\begin{aligned}
=\frac{i}{2} \int_{0}^{\infty} d \lambda \frac{d^{4} p}{(2 \pi)^{4}} & \times \exp \left\{-i p\left(x_{f}-x_{i}\right)+\frac{i \lambda\left(p^{2}-m^{2}\right)}{2}\right. \\
& +\frac{i g^{2}}{2 p k} \int_{k x_{i}}^{k x_{f}} \frac{A_{\mu}^{a} A_{a}^{\mu}}{2 N} d \varphi \\
& -\frac{i g}{p k}\left[\int_{k x_{i}}^{k x_{f}}\left(p^{\mu} A_{\mu}^{a} T_{a}\right) d \varphi\right. \\
& \left.\left.+i\left(\frac{k \gamma}{2}\right) \gamma^{\mu}\left(A_{\mu}^{a}\left(x_{f}\right)-A_{\mu}^{a}\left(x_{i}\right)\right) T_{a}\right]\right\} .
\end{aligned}
$$

So, Green's function relative to our problem is after derivation as follows:

$$
\begin{aligned}
S=\frac{i}{2} \int_{0}^{\infty} d \lambda \int \frac{d^{4} p}{(2 \pi)^{4}} & \left\{(\widehat{p}+m)\left[1+g \frac{\widehat{k}}{2 k p}\left(\widehat{A}^{a}\left(x_{f}\right)-\widehat{A}^{a}\left(x_{i}\right)\right) T_{a}\right]\right. \\
\times & \frac{g}{k p} \widehat{k}\left(p A\left(x_{f}\right) T\right)-g\left(\widehat{A}\left(x_{f}\right) T\right) \\
& \left.-g^{2} \widehat{k} \frac{A\left(x_{f}\right) A\left(x_{i}\right)}{2 N(k p)}\right\} \\
\times & \exp \left\{-i p\left(x_{f}-x_{i}\right)+\frac{i \lambda}{2}\left(p^{2}-m^{2}\right)\right. \\
& +\frac{i g^{2}}{2 p k} \int_{k x_{i}}^{k x_{f}} \frac{A_{\mu}^{a} A_{a}^{\mu}}{2 N} d \varphi \\
& \left.-\frac{i}{p k} g \int_{k x_{i}}^{k x_{f}}\left(p^{\mu} A_{\mu}^{a} T_{a}\right) d \varphi\right\} .
\end{aligned}
$$

It can be noted that this expression is not symmetrical, and therefore it is not possible at this level to extract wave functions. To render symmetric, let us use the following relation:

$$
\widehat{a} \widehat{b}+\widehat{b} \widehat{a}=2 a b,
$$

where $\widehat{a}=\gamma^{\mu} a_{\mu}$.

$$
p^{\mu} \longrightarrow-p^{\mu}+k^{\mu} p_{\varphi}
$$


After some manipulations, $S$ becomes

$$
\begin{aligned}
S= & -\int \frac{d^{4} p}{(2 \pi)^{4}}\left\{1+\frac{g \widehat{k}}{2 k p}\left(\widehat{A}\left(x_{f}\right) T\right)\right\} \frac{(\widehat{p}+m)}{\left(p^{2}-m^{2}\right)} \\
& \times\left\{1-\frac{g \hat{k}}{2 k p}\left(\widehat{A}\left(x_{i}\right) T\right)\right\} \\
& \times \exp \left\{-i p\left(x_{f}-x_{i}\right)+\frac{i g^{2}}{2 p k} \int_{k x_{i}}^{k x_{f}} \frac{A_{\mu}^{a} A_{a}^{\mu}}{2 N} d \varphi\right. \\
& \left.-\frac{i}{p k} g \int_{k x_{i}}^{k x_{f}}\left(p^{\mu} A_{\mu}^{a} T_{a}\right) d \varphi\right\}
\end{aligned}
$$

that is, $S$ is totally symmetric. Finally, a simple integration over $p^{0}$ leads to the following form:

$$
\begin{aligned}
S\left(x_{f}, x_{i}\right)= & -i \theta\left(\tau_{f}-\tau_{i}\right) \sum_{\alpha \pm \sigma} \int d^{3} p \Psi_{\alpha, \sigma, p}^{+}\left(x_{f}\right) \bar{\Psi}_{\alpha, \sigma, p}^{+}\left(x_{i}\right) \\
& -i \theta\left(\tau_{i}-\tau_{f}\right) \sum_{\alpha \pm \sigma} \int d^{3} p \Psi_{\alpha, \sigma, p}^{-}\left(x_{f}\right) \bar{\Psi}_{\alpha, \sigma, p}^{-}\left(x_{i}\right),
\end{aligned}
$$

where the wave functions (normalized) describing the motion of Dirac particle in interaction with external nonAbelian SU(N) gauge field are

$$
\begin{aligned}
\Psi_{\alpha, \sigma, p}^{+}(x)= & \frac{1}{(2 \pi)^{3 / 2}}\left(\frac{m}{p^{0}}\right)^{1 / 2} \\
& \times \exp \left[-i p x+\frac{i g^{2}}{2 p k} \int_{0}^{k x}\left(\frac{A_{\mu}^{a} A_{a}^{\mu}}{2 N}\right) d \varphi\right] \\
& \times \exp \left[-\frac{i g}{k p} \int_{0}^{k x}(p A T) d \varphi+\frac{g(k \gamma)}{2 k p}(\gamma A T)\right] \\
& \times u_{\sigma}(p) w_{\alpha},
\end{aligned}
$$

with

$$
\begin{aligned}
& \sum_{ \pm \sigma} u_{\sigma}(p) \bar{u}_{\sigma}(p)=\frac{(\widehat{p}+m)}{2 m}, \\
& \sum_{ \pm \sigma} v_{\sigma}(p) \bar{v}_{\sigma}(p)=\frac{(-\widehat{p}+m)}{2 m},
\end{aligned}
$$

the projectors on positive and negative states energy and $w_{\alpha}, w_{\beta}$ the elements of the line $w^{+}$and the column $w$ (respectively) such as

$$
w^{+} w=I
$$

or also

$$
w_{\alpha}^{+} w_{\beta}=\delta_{\alpha \beta}
$$

Finally, the wave function can be written in the form given in $[8]$

$$
\begin{aligned}
& \Psi_{\alpha, \sigma, p}^{+}(x) \\
& =\frac{1}{(2 \pi)^{3 / 2}}\left(\frac{m}{p^{0}}\right)^{1 / 2} \\
& \times \exp \left[-i p x+\frac{i g^{2}}{2 p k} \frac{1}{2 N} \int_{0}^{k x}\left(A_{\mu}^{a} A_{a}^{\mu}\right) d \varphi\right] \\
& \times \cos \theta\left\{\left(1-i g T_{a} \frac{\tan \theta}{\theta(p k)} \int_{0}^{k x}\left(p^{\mu} A_{\mu}^{a}\left(\varphi^{\prime \prime}\right)\right) d \varphi^{\prime \prime}\right)\right. \\
& +\frac{g\left(k_{\mu} \gamma^{\mu}\right)\left(\gamma^{\mu} A_{\mu}^{a}\right)}{2 k p} \\
& \times\left[\frac{\tan \theta}{\theta} T_{a}+\frac{g}{p k} \frac{1}{2 N} \int_{0}^{k x} d \varphi^{\prime \prime}\left(p^{\mu} A_{\mu}^{a}\left(\varphi^{\prime \prime}\right)\right)\right. \\
& \times\left(-i \frac{\tan \theta}{\theta}+\frac{g}{p k} \frac{\theta-\tan \theta}{\theta^{3}} T_{b}\right. \\
& \left.\left.\left.\times \int_{0}^{k x} d \varphi^{\prime \prime}\left(p^{\mu} A_{\mu}^{b}\left(\varphi^{\prime \prime}\right)\right)\right)\right]\right\} \\
& \times u_{\sigma}(p) w_{\alpha}
\end{aligned}
$$

with

$$
\begin{aligned}
\theta=\left[\left(\frac{g}{k p}\right)\left(\frac{1}{2 N}\right)^{1 / 2}\right] & {\left[\left(\int_{0}^{k x}\left(p^{\mu} A_{\mu}^{a}\left(\varphi^{\prime}\right)\right) d \varphi^{\prime}\right)\right.} \\
& \left.\times\left(\int_{0}^{k x}\left(p^{\mu} A_{\mu}^{a}\left(\varphi^{\prime \prime}\right)\right) d \varphi^{\prime \prime}\right)\right]^{1 / 2} .
\end{aligned}
$$

\section{Conclusion}

In the present work, by using the formalism of Alexandrou et al., we have determined the wave function for a Dirac particle moving under the action of a field non-Abelian. Thanks to the introduction of two constraints, $\varphi=k x$ (related to space) and Grassmannian $\eta=k \psi$ (related to Dirac matrices), it is appeared Dirac functions; that is, the paths having simple equations are selected and it is these paths which have contributed to the determination of the propagator. From the obtained analytical expression of the propagator, the wave functions extracted are found to be the same as those obtained by direct resolution of the Dirac equation.

Finally it can be shown in appendix that the selected paths which have played an important role in the determination of propagator or Green function can be also obtained from the equations of classical mechanics. 


\section{Appendix}

Action, in Global Approach, is

$$
\begin{aligned}
\mathscr{A}=\int_{0}^{\lambda}\{p \dot{x}+i \zeta \dot{\zeta}+i \psi \dot{\psi} \\
+\frac{1}{2}\left(p^{2}-m^{2}+g^{2} \frac{A_{\mu}^{a} A_{a}^{\mu}}{2 N}\right. \\
+2 i g\left(p^{\mu} A_{\mu}^{a} f_{a p}^{b} \zeta_{b} \zeta^{p}\right) \\
\left.\left.+4 g(k \psi)\left(\psi^{\mu} \dot{A}_{\mu}^{a} f_{a p}^{b} \zeta_{b} \zeta^{p}\right)\right)\right\} d \tau,
\end{aligned}
$$

and the equations of motion (classical) are, respectively,

$$
\begin{gathered}
\frac{\delta \mathscr{A}}{\delta x^{\rho}}=-\dot{p}_{\rho}+\frac{1}{2} k_{\rho}\left(g^{2} \frac{\dot{A}_{\mu}^{a} A_{a}^{\mu}}{N}+2 i g\left(p^{\mu} \dot{A}_{\mu}^{a} f_{a p}^{b} \zeta_{b} \zeta^{p}\right)\right. \\
\left.+4 g(k \psi)\left(\psi^{\mu} \ddot{A}_{\mu}^{a} f_{a p}^{b} \zeta_{b} \zeta^{p}\right)\right) \\
\frac{\delta \mathscr{A}}{\delta p^{\rho}}=\dot{x}_{\rho}+p_{\rho}+i g\left(A_{\rho}^{a} f_{a p}^{b} \zeta_{b} \zeta^{p}\right) \\
\frac{\delta \mathscr{A}}{\delta \psi^{\rho}}=2 i \dot{\psi}_{\rho}+2 g k_{\rho}\left(\psi^{\mu} \dot{A}_{\mu}^{a} f_{a p}^{b} \zeta_{b} \zeta^{p}\right)-2 g(k \psi)\left(\dot{A}_{\rho}^{a} f_{a p}^{b} \zeta_{b} \zeta^{p}\right), \\
\frac{\delta \mathscr{A}}{\delta \zeta^{\rho}}=2 i \dot{\zeta}_{\rho}-4 i g\left(p^{\mu} A_{\mu}^{a} f_{a \rho}^{p} \zeta_{p}\right)-8 g(k \psi)\left(\psi^{\mu} \dot{A}_{\mu}^{a} f_{a \rho}^{b} \zeta_{b}\right) .
\end{gathered}
$$

By multiplying (A.3), (A.4) by $k$ and thanks to the properties of the Volkov field, we obtain the following relations:

$$
\begin{gathered}
k \dot{x}=-p k, \quad \frac{d \varphi}{d \tau}=-p k, \\
k \psi=C^{s t} .
\end{gathered}
$$

Thus, the selected equations appeared in the arguments of Dirac functions $\delta$ are exactly the classical equations.

To prove that $p_{\mu}=c^{t e}$, we use (A.2)

$$
\begin{gathered}
\dot{p}_{\rho}-\frac{e k_{\rho}}{2}\left(2 i g\left(p^{\mu} \dot{A}_{\mu}^{a} f_{a p}^{b} \zeta_{b} \zeta^{p}\right)+4 g(k \psi)\left(\psi^{\mu} \ddot{A}_{\mu}^{a} f_{a p}^{b} \zeta_{b} \zeta^{p}\right)\right. \\
\left.+g^{2} \frac{\dot{A}_{\mu}^{a} A_{a}^{\mu}}{N}\right)=0,
\end{gathered}
$$

and let us introduce the total derivative $d / d \tau$ in the 2 nd and 3rd terms,

$$
\begin{aligned}
2 i g\left(p^{\mu} \dot{A}_{\mu}^{a} f_{a p}^{b} \zeta_{b} \zeta^{p}\right) & \\
= & -\frac{2 i g}{p k} \frac{d}{d \tau}\left(p^{\mu} A_{\mu}^{a} f_{a p}^{b} \zeta_{b} \zeta^{p}\right) \\
& +\frac{8 g^{2}}{p k}(k \psi)\left(p^{\mu} A_{\mu}^{a} f_{a p}^{b} \zeta^{p}\left(\psi^{\nu} \dot{A}_{\nu}^{c} f_{c b}^{l} \zeta_{l}\right)\right), \\
4 g(k \psi)\left(\psi^{\mu} \ddot{A}_{\mu}^{a} f_{a p}^{b} \zeta_{b} \zeta^{p}\right) & -\frac{4 g}{p k} \frac{d}{d \tau}(k \psi)\left(\psi^{\mu} \dot{A}_{\mu}^{a} f_{a p}^{b} \zeta_{b} \zeta^{p}\right) \\
& -\frac{8 g^{2}}{p k}(k \psi)\left(\psi^{\mu} \dot{A}_{\mu}^{a} f_{a p}^{b} \zeta^{p}\left(p^{\nu} A_{\nu}^{c} f_{c b}^{l} \zeta_{l}\right)\right) ;
\end{aligned}
$$

then (A.2) becomes

$$
\begin{aligned}
0=\frac{d}{d \tau}\left[p_{\rho}+\frac{1}{2 p k} k_{\rho}(\right. & g^{2} \frac{A_{\mu}^{a} A_{a}^{\mu}}{2 N}+2 i g\left(p^{\mu} A_{\mu}^{a} f_{a p}^{b} \zeta_{b} \zeta^{p}\right) \\
& \left.\left.+4 g(k \psi)\left(\psi^{\mu} \dot{A}_{\mu}^{a} f_{a p}^{b} \zeta_{b} \zeta^{p}\right)\right)\right]
\end{aligned}
$$

that is, we have

$$
\begin{aligned}
P_{\rho}=p_{\rho}+\frac{k_{\rho}}{2 p k}( & g^{2} \frac{A_{\mu}^{a} A_{a}^{\mu}}{2 N}+2 i g\left(p A^{a} f_{a p}^{b} \zeta_{b} \zeta^{p}\right) \\
& \left.+4 g(k \psi)\left(\psi \dot{A}^{a} f_{a p}^{b} \zeta_{b} \zeta^{p}\right)\right)
\end{aligned}
$$$$
=C^{t e} \text {. }
$$

It can be noticed that $k P=k p$ and that the shift $p_{\rho} \rightarrow$ $P_{\rho}=p_{\rho}+(1 / 2 p k) k_{\rho}(\cdot)$ does not affect the calculation of $G$.

\section{Conflict of Interests}

The authors declare that there is no conflict of interests regarding the publication of this paper.

\section{References}

[1] E. S. Fradkin and D. M. Gitman, "Path-integral representation for the relativistic particle propagators and BFV quantization," Physical Review D, vol. 44, no. 10, pp. 3230-3236, 1991.

[2] S. Zeggari, T. Boudjedaa, and L. Chetouani, "Path integral for Dirac particle in plane wave field," Physica Scripta, vol. 64, no. 4, pp. 285-291, 2001.

[3] N. Boudiaf, T. Boudjedaa, and L. Chetouani, "Exact propagators for Dirac particle with anomalous magnetic moment in a plane wave field," European Physical Journal C, vol. 22, pp. 593-600, 2001.

[4] N. Boudiaf, A. Merdaci, and L. Chetouani, "Path integral solution for Dirac particle in a constant electric field," Journal of Physics A, vol. 42, no. 1, Article ID 015303, 2009. 
[5] A. Merdaci, N. Boudiaf, and L. Chetouani, "Dirac particle in a constant magnetic field: path integral treatment," Zeitschrift fur Naturforschung A, vol. 63, no. 5-6, pp. 283-290, 2008.

[6] S. Haouat and L. Chetouani, "Covariant path integral for the Dirac equation with pseudoscalar potentials," Journal of Physics A, vol. 40, no. 6, pp. 1349-1359, 2007.

[7] E. S. Fradkin, D. M. Gitman, and S. M. Shvartsman, Quantum Electrodynamics Whith Unstable Vacuum, Springer, Berlin, Germany, 1991.

[8] A. V. Koshelkin, "Solution of Dirac equation in external YangMills gauge field," Physics Letters B, vol. 683, no. 2-3, pp. 205-210, 2010.

[9] C. Alexandrou, R. Rosenfelder, and A. W. Schreiber, "Worldline path integral for the massive Dirac propagator: a fourdimensional approach," Physical Review A, vol. 59, no. 3, pp. 1762-1776, 1999. 

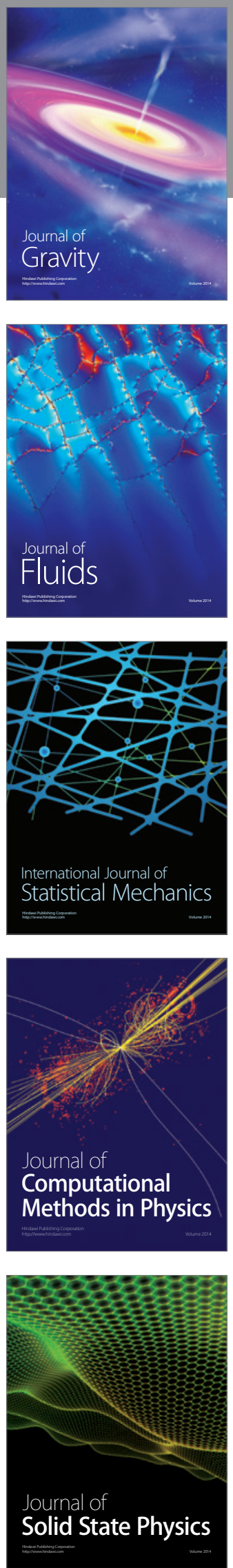

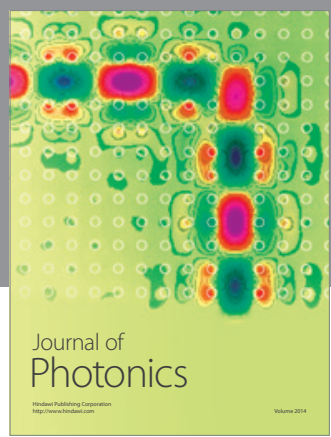

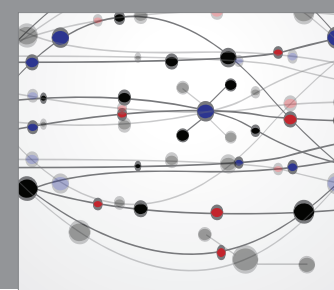

The Scientific World Journal

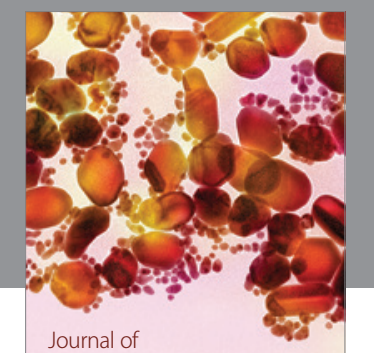

Soft Matter
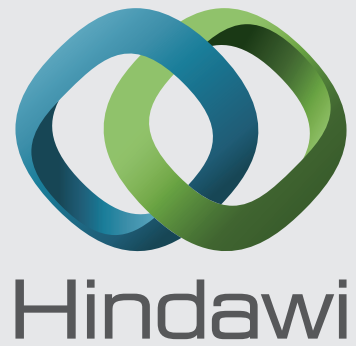

Submit your manuscripts at

http://www.hindawi.com
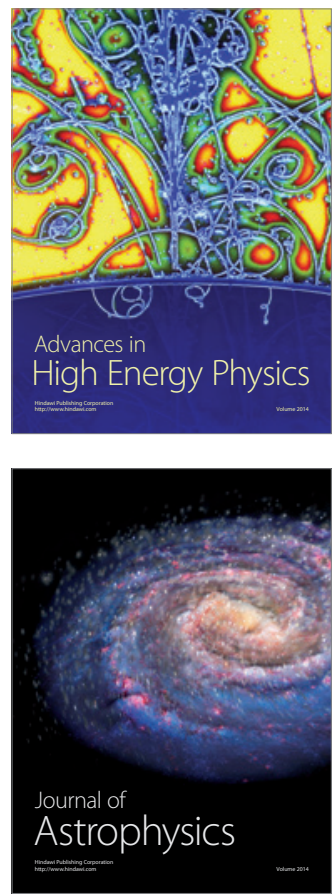
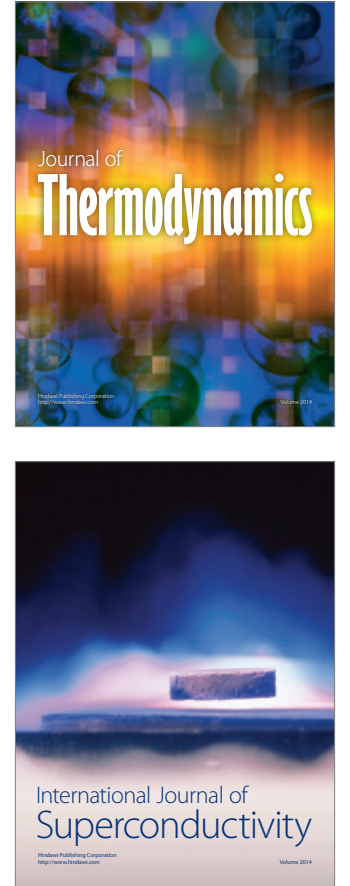
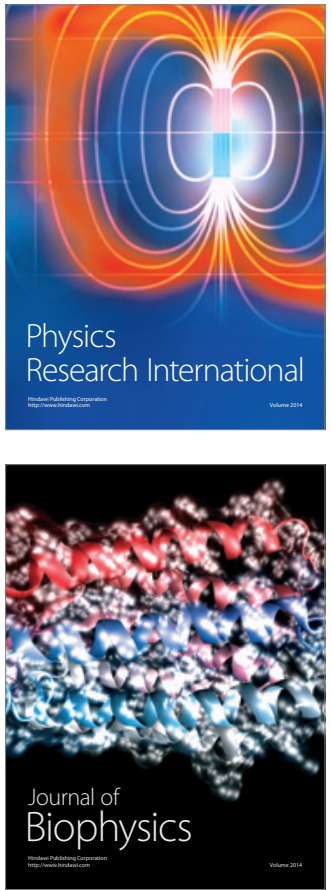
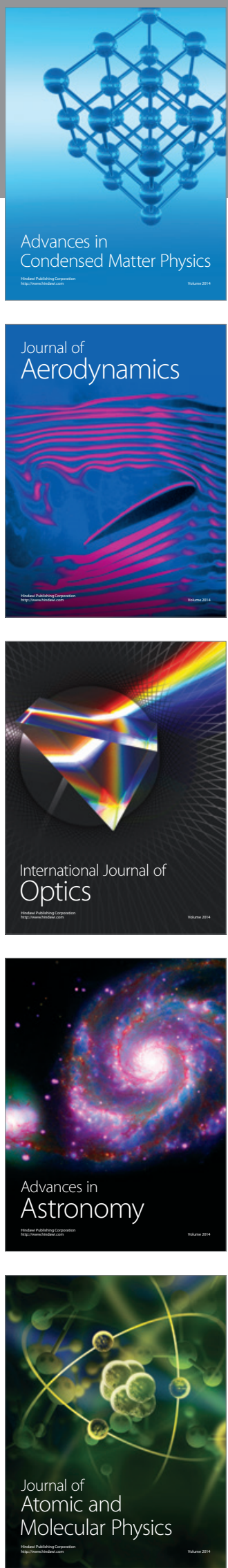